\title{
LEAD THIOCYANATE.
}

\author{
BY R, D. HALL.
}

Received March 2I, rgoz.

NORMaL lead thiocyanate is described by Liebig ${ }^{1}$ as a yellow precipitate formed by the action of potassium thiocyanate on lead acetate. The salt, on standing for some time undisturbed, separates as large glistening crystals which are almost insoluble in cold water, but are decomposed by boiling with the formation of a yellow, completely insoluble basic salt. Liebig assigned the formula $\mathrm{Pb}(\mathrm{CNS})_{2}$ to the normal salt which formula was established by analysis. The analysis of the basic salt obtained by him is in better agreement with the formula $\mathrm{PbO} \cdot \mathrm{Pb}(\mathrm{CNS})_{2}$ than with $\mathrm{Pb}(\mathrm{OH})$ (CNS), the formula given in the literature.

In the preparation of lead thiocyanate from pure lead nitrate and potassium thiocyanate, a compound was obtained which differed somewhat from the salt described by Liebig. The crystals were monoclinic like Liebig's but were white and appeared either in large transparent crystals or separated as a feathery mass. Analysis showed these crystals to contain 63.98 per cent. of lead while the formula $\mathrm{Pb}(\mathrm{CNS})_{2}$ requires 64.09 per cent. They are completely soluble in hot water and crystallize from solution without suffering decomposition. 3.3 parts of lead thiocyanate dissolve in I00 parts of boiling water, while at $23^{\circ} \mathrm{C}$. 0.69 of a part dissolves. Analysis of the recrystallized salt showed 64.03 per cent. of lead.

The crystals were boiled with water for several hours and showed no such decomposition as is described by I.iebig, who mentions that under these conditions an insoluble basic salt is formed while at the same time the solution becomes acid. The basic salt can be obtained by the addition of potassium thiocyanate to a solution of basic lead acetate and appears as a white precipitate. The salt prepared in this way shows a lead content of 73.3 per cent. of lead. That required for $\mathrm{Pb}(\mathrm{OH})(\mathrm{CNS})$ is 73.2 per cent., while the salt obtained by $L_{4}$ iebig by the addition of ammonia to a mixture of normal lead acetate and potassium thiocyanate or

1 Pogg. Ann., 25, 546. 
by the decomposition of the normal lead thiocyanate by boiling water, contained 74.96 per cent. of lead, corresponding more nearly to the compound $\mathrm{PbO} . \mathrm{Pb}(\mathrm{CNS})_{2}$ which requires 75.7 per cent. of lead. Basic lead thiocyanate is sparingly soluble in boiling water, and crystallizes from hot solutions in small acicular crystals. It is less soluble in water than chloride and more soluble than chromate of lead.

In seeking to ascertain the cause of the yellow discoloration of lead thiocyanate as obtained by Liebig, an investigation was made of the effect of the presence of other salts on the character of the precipitate. It was found that, if the lead solution contains iron, mercury or bismuth salts, the product formed by the addition of potassium thiocyanate is yellow. If a considerable amount of iron is in the solution from which lead thiocyanate is precipitated, the precipitate is yellow with a decided pink cast, while the liquid shows the intense red coloration of ferric thiocyanate. The substance thus obtained, when washed and boiled with water, leaves a residue which resembles ferric hydroxide. When mercuric salts are present in the solution from which lead thiocyanate is obtained, the latter is greenish yellow and is completely soluble in hot water.

The effect of bismuth in the solution has been carefully studied. Basic bismuth thiocyanate was prepared by treating a solution of bismuth oxide in acetic acid with potassium thiocyanate. The white basic salt separates out on heating, while the mother-liquor, when concentrated, yields the finely divided red normal salt. When lead acetate solution contains a small quantity of bismuth acetate, the precipitate which appears by the addition of potassium thiocyanate, comes down slowly and is yellow and crystalline. Analysis of the yellow salt showed 63.8 and 63.95 per cent. of lead, while the normal salt contains 64.09 per cent. While the per cent. of lead is only slightly less than that of the theoretical, the crystals were distinctly yellow, due to the bismuth which they contained. A distinct reaction for bismuth was obtained, after removing the lead as sulphate, by the addition of an alkaline solution of stannous chloride. Moreover all but a very small quantity of the salt dissolved in boiling water. When the solution was filtered, the clear liquid showed an acid reaction and on cooling white crystals appeared. The residue gave a decided reaction for bismuth and 
none whatever for lead, showing the bismuth salt to be contained in the lead thiocyanate crystals as impurities which can be removed by crystallization.

Normal solutions of lead nitrate and bismuth nitrate, mixed in various proportions, were precipitated with potassium thiocyanate. The precipitate in each case was filtered, washed rapidly with water, then with alcohol, and dried in an air-bath. The substances thus obtained varied in color from a light yellow to a dark red, depending on the ratio of bismuth to lead in the original solution; the more bismuth the darker was the resulting salt. As nitric acid readily oxidlizes lead thiocyanate to sulphate, it is necessary to immediately remove the salt from the liquor by filtration. The precipitates contained varying amounts of bismuth from a trace up to from 6 to 7 per cent. No relation could be determined between the amount of lead thiocyanate and bismuth thiocyanate in the precipitate pointing to the formation of a definite double salt, neither was there any noticeable relation between the amounts of bismuth and lead in the solution to that of bismuth thiocyanate and lead thiocyanate in the precipitate, except the general one that the more bismuth in the solution the more in the precipitate.

A solution which contained Io cc. of normal bismuth nitrate and Ioo cc. of normal lead nitrate gave, with potassium thiocyanate, a precipitate that contained 6r.7 per cent. of lead and 2.05 per cent. of bismuth, which is about the proportion of I part of bismuth to 30 parts of lead, while the ratio in the solution was I part of bismuth to Io of lead. Where the ratio in the solution was I of bismuth to 2 of lead, the salt obtained by the addition of potassium thiocyanate contained 2.8 per cent. of bismuth and 60.8 per cent. of lead, or in about the proportion of I part of bismuth to 22 parts of lead. The precipitate from a solution containing five times as much bismuth as lead contained nine times as much lead as bismuth, analysis showing 56.4 per cent. of lead and 6.3 per cent. of bismuth.

Cold water slowly decomposes the yellow salt thus formed, washing away the lead salt and leaving the bismuth. On boiling, all of the lead salt goes into solution leaving a nearly white gelatinous residue, while the liquid becomes acid. Extraction with water does not give a complete separation, as some bismuth is ex- 
tracted along with lead. Repeated crystallization will entirely remove the yellow color of the lead thiocyanate. It requires only a trace of bismuth in the solution to give a decided yellow cast to the precipitate, yet a considerable quantity is necessary before enough bismuth is present to appear as a residue when dissolved in boiling water.

Lead thiocyanate is white when pure but is readily contaminated by the presence of foreign salts, especially bismuth, in the solution from which it is precipitated. It is entirely soluble in hot water crystallizing out unchanged on cooling, and can be purified by repeated crystallization. Basic lead thiocyanate can not be prepared. by boiling the normal salt with water, but must be prepared from a solution of a basic salt such as the acetate.

UNIVERSITY OF WISCONSIN,

MADISON, WISCONSIN.

[CONTRIBUTION FROM THE JOHN HARRISON LABORATORY OF ChEMISTRY, No. 63.]

AMTONIUR VANADICOPHOSPHOTUNGSTATE.

BY EDGAR F. SMITH AND FRANZ F. EXNER.

Received March 66, rg02.

During the summer months of Igor large quantities of wolframite were examined by us for the purpose of ascertaining just what minute constituents were present in it, which might possibly contaminate the tungstic acid derived from this source. That such impurities existed in tungstic acid seemed very probable as the numerous attempts to fix the atomic weight of tungsten, by reduction of the oxide in hydrogen and subsequent oxidation of the tungsten metal, gave unsatisfactory results. The difference between the maximum and minimum values seemed too great to be attributed entirely to the inefficiency of the method so it was determined to seek for impurities, some hint as to the nature of which it was thought could be best realized by taking into study large quantities of the starting-material-wolframite, from Lawrence County, South Dakota.

The finely divided material was decomposed by prolonged digestion with aqua regia. The hydrated tungstic acid, after filtration and thorough washing, was digested with ammonia water. 